\title{
Die Grenzen der Demokratie. Politische Auseinandersetzungen um Rechtsextremismus im österreichischen Nationalrat
}

\author{
Matthias Falter \\ Baden-Baden / Wien 2019 (= Interdisziplinäre Antisemitismusforschung, Bd. 10): Nomos / facultas, 289 S.
}

Heinz P. Wassermann

FH JOANNEUM, Institut Journalismus und Public Relations, Austria

E-Mail: heinz.wassermann@fh-joanneum.at; Twitter: @heinzwassermann

In seiner, von Eva Kreisky betreuten, 2017 eingereichten und mittlerweile in Buchform vorliegenden politikwissenschaftlichen Dissertation bearbeitet Matthias Falter mit den Stenographischen Protokollen des Österreichischen Nationalrates einen bislang allenfalls gelegentlich verwendeten, alles in allem - wie sich zeigt - jedoch sträflich vernachlässigten Quellenbestand. Die auf der „schriftlichen Verfahrensöffentlichkeit“ (Schefbeck, 2006, 160) des Nationalrates basierende Arbeit füllt eine echte, wenn auch zeitlich begrenzt ausgewertete, Forschungslücke. Die Debatten wurden auf zwei Ebenen geführt: einerseits als „Verhandlung über Rechtsextremismus“, andererseits als „Auseinandersetzung mit extrem rechten Politiken." (249)

Untersucht wird der Zeitraum 1999 bis 2013 bzw. die XXI. bis zur XXIV. Gesetzgebungsperiode. In diesem Abschnitt fanden 574 Sitzungen statt, wovon 224 , das sind bemerkenswerte 39 Prozent (vgl. I27), auswertungsbzw. themenrelevant waren.

Das Buch besteht aus einem theoretischen (23-I26) und einem empirisch-analytischen (I27-247) Teil, wobei die Ausführungen zum Framing (40-44) und zum Untersuchungszeitraum (I2I-I26) auch in den zweiten Teil gepasst hätten. Dadurch, dass die zwei Teile inhaltlich nicht streng voneinander getrennt (möglicherweise auch nicht exakt voneinander abgrenzbar) sind, kommt es zu einigen inhaltlichen Redundanzen.

Der Arbeit liegt ein zwar zeitlich „angegrauter“, nichtsdestotrotz über weite Strecken noch immer gültiger und verwendbarer, an Willibald I. Holzer (vgl. Holzer 1993) orientierter, „breiter“ Rechtsextremismusbegriff zugrunde. Rechtsextremismus wird sowohl als politische Ideologie als auch als antidemokratisches und/oder gewaltbereites Handeln sowie als anpassungsfähige, flexible und dementsprechend "modernisierte $/$ „modernisierungsfähige" politische Strategie verstanden. Die gemeinsame Klammer ist das Postulat („natürlicher“) Ungleichheit, das ein Spektrum von Antiliberalismus über Antisemitismus bis hin zu Rassismus und Sexismus abdeckt.

Sofern es den österreichischen Parlamentarismus bzw. die dort via Wahlen legitimierten Parteien betrifft, nimmt die "rechtspopulistische“ und in "Aspekten (...) rechtsextreme" (II2) FPÖ wenig überraschend eine zentrale Rolle ein. Allerdings, und das ist eine der (potentiellen) Hauptstärken des Buches, „wäre [es] jedoch falsch, den Blickwinkel nur auf die FPÖ zu richten" (96), womit Falter zum einen die Anschlussfähigkeit (extrem) rechter/rechtsextremer Positionen, zum anderen die Diskurs- und Deutungsverschiebung des Sagbaren in Richtung politische "Mitte" meint. Trotzdem wurde „Rechtsextremismus (...) im österreichischen Nationalrat meist in Zusammenhang mit [der] FPÖ“ bzw. mit FPÖ-Politikern, wie die Wahlen von Thomas Prinzhorn (1999) und von Martin Graf (2008) zum Zweiten bzw. zum Dritten Nationalratspräsidenten, die Wahl Peter Fichtenbauers zum Volksanwalt (2OI3) sowie die historischen Unsäglichkeiten von Ewald Stadler (2002), Siegfried Kampl oder John Gudenus 2005 „debattiert“" (174).

Im Zuge der empirischen Untersuchungen arbeitet der Verfasser insgesamt vier Frames heraus, die sich allerdings in den parlamentarischen Debatten nicht nur nicht selten, sondern regelmäßig überlagerten.

Als historische Folie des geschichtspolitischen Frames (18I-195) dient der Nationalsozialismus bzw. das (gegenwärtige) Verhältnis zum (historischen) Nationalsozi- 
alismus. In diesem Kontext zeigen sich drei Argumentationen: eine antifaschistische (Die Grünen und SPÖ), eine antinazistische (ÖVP) und eine antitotalitäre (FPÖ). Der ordnungspolitische Frame (195-215), der in den Debatten häufig mit dem eben Erwähnten verknüpft und für den Autor eine „entpolitisierende Deutung“ (215) von Rechtsextremismus ist, deutet Rechtsextremismus „als Abweichung von der öffentlichen Ordnung und (...) der politischen Normalität", wodurch er zur "Gefahr für die demokratischen Institutionen" (I95)wird und darüber hinaus eine strafrechtliche Dimension aufweist. Der dritte, von Falter als identitätspolitisch (215-230) kategorisierte Frame, findet sich häufig im Konnex mit außenpolitischen Debatten, konkret, wenn Rechtsextremismus dazu angetan ist, dem internationalen Ansehen Österreichs zu schaden. Auffallend ist der Umstand, dass er häufig in Richtung Patriotismusdebatte abgleitet. Der demokratiepolitische Frame (230-247) begreift Rechtsextremismus zum einen als das "Gegenteil von Demokratie“ (23I), ist aber zum anderen auch ein „Streit über die konkrete Ausgestaltung von Demokratie selbst [...], insbesondere dann, wenn die demokratische Position einzelner $\mathrm{Ab}$ geordneter oder Parteien [...] in Frage gestellt wird oder verteidigt werden muss" (230-23I). Wie der Geschichtspolitische ist auch dieser Frame "als abstrakter Konsens“ (23I) konsensfähig, sprich elastisch genug, um auch extrem rechte bzw. rechtsextreme Position(ierung)en in den demokratischen Konsens zu integrieren.

Eine Stärke des Buches liegt in der konsequenten und vorbildlichen Verknüpfung von „politischer Theorie“ mit "empirischer Politikforschung" (250) und der Auswertung eines neuen Quellenbestandes, der Ausgangspunkt für weitere Forschungsarbeiten sein sollte.

Last but not least deutet die äußerst lesenswerte und akribisch genau gearbeitete Publikation auch eine Verschiebung österreichischer Rechtsextremismusforschung an. War diese über Jahrzehnte beim Dokumentationsarchiv des Österreichischen Widerstandes quasimonopolisiert, ist eine Verschiebung bzw. Pluralisierung - man denke beispielsweise an die Online-Watchdogs „FPÖ Fails“, „FPÖ Watch“ und „stopptdierechten“ oder an FIPU („Forschungsgruppe Ideologie und Politiken der Ungleichheit"), als dessen Mitglied der Verfasser auf deren Website aufscheint - bemerkbar. Dieser grundsätzlich erfreuliche Umstand ändert jedoch nichts am weiterhin geringen Maß an Institutionalisierung und somit an Forschungskontinuität.

\section{Literatur}

Holzer, Willibald I. (1993), Rechtsextremismus - Konturen, Definitionsmerkmale und Erklärungsansätze, in: Dokumentationsarchiv des Österreichischen Widerstan- des (Hrsg.), Handbuch des österreichischen Rechtsextremismus, 2. Auflage, Wien: Deuticke, II-96.

Schefbeck, Günther (2006), Das Parlament, in: Dachs, Herbert/Peter Gerlich/Herbert Gottweis/Helmut Kramer/ Volkmar Lauber/Wolfgang C. Müller/Emmerich Tálos (Hrsg.), Politik in Österreich. Das Handbuch, Wien: Manz, I39-167. 